\title{
Improvement in cognitive function after surgery for low-grade glioma
}

\author{
Ori Barzilai, MD, Shlomit Ben Moshe, MA, Razi Sitt, MSW, Gal Sela, MA, Ben Shofty, MD, and \\ Zvi Ram, MD
}

Department of Neurosurgery, Tel Aviv Sourasky Medical Center, Tel Aviv University, Tel Aviv, Israel

OBJECTIVE Cognition is a key component in health-related quality of life (HRQoL) and is currently incorporated as a major parameter of outcome assessment in patients treated for brain tumors. The effect of surgery on cognition and HRQoL remains debatable. The authors investigated the impact of resection of low-grade gliomas (LGGs) on cognition and the correlation with various histopathological markers.

METHODS A retrospective analysis of patients with LGG who underwent craniotomy for tumor resection at a single institution between 2010 and 2014 was conducted. Of 192 who underwent resective surgery for LGG during this period, 49 had complete pre- and postoperative neurocognitive evaluations and were included in the analysis. These patients completed a full battery of neurocognitive tests (memory, language, attention and working memory, visuomotor organization, and executive functions) pre- and postoperatively. Tumor and surgical characteristics were analyzed, including volumetric measurements and histopathological markers (IDH, p53, GFAP).

RESULTS Postoperatively, significant improvement was found in memory and executive functions. A subgroup analysis of patients with dominant-side tumors, most of whom underwent intraoperative awake mapping, revealed significant improvement in the same domains. Patients whose tumors were on the nondominant side displayed significant improvement only in memory functions. Positive staining for p53 testing was associated with improved language function and greater extent of resection in dominant-side tumors. GFAP positivity was associated with improved memory in patients whose tumors were on the nondominant side. No correlation was found between cognitive outcome and preoperative tumor volume, residual volume, extent of resection, or IDH1 status.

CONCLUSIONS Resection of LGG significantly improves memory and executive function and thus is likely to improve functional outcome in addition to providing oncological benefit. GFAP and pP53 positivity could possibly be associated with improved cognitive outcome. These data support early, aggressive, surgical treatment of LGG.

https://thejns.org/doi/abs/10.3171/2017.9.JNS17658

KEY WORDS cognition; HRQoL; tumor; craniotomy; p53; GFAP; IDH; oncology

$\mathrm{C}$ OGNITIVE deficits are frequently present at the time of brain tumor diagnosis but are often not recognized. ${ }^{38,43,45,53}$ Neurocognition is now considered a crucial function for maintaining quality of life and in selecting treatment options in patients with glial tumors. ${ }^{21}$ Cognitive status has also been linked to the survival of glioma patients ${ }^{6}$ and is a key component in patient-related outcome scores. ${ }^{46}$

Surgery for glial tumors has been shown to improve rates of progression-free survival and overall survival in patients with low- and high-grade gliomas, and maximal resection of these tumors is becoming the standard of care. ${ }^{31,35,39}$ Surgery as a first treatment choice for newly diagnosed low-grade glioma (LGG) is becoming more common, as it also improves seizure control and reduces the risk of malignant transformation. ${ }^{18,36,37}$ Since most patients with gliomas, including diffuse low-grade tumors, cannot be cured but may enjoy long periods of disease-free survival, ${ }^{1}$ the palliation of symptoms and maintenance or improvement of physical and cognitive functions are important goals of treatment. Thus, there is currently increased interest in functional outcomes alongside oncological out-

ABBREVIATIONS EOR = extent of tumor resection; fMRI = functional MRI; FWE = family-wise error; HRQoL = health-related quality of life; LGG = low-grade glioma. SUBMITTED March 14, 2017. ACCEPTED September 5, 2017.

INCLUDE WHEN CITING Published online March 23, 2018; DOI: 10.3171/2017.9.JNS17658. 
comes in this patient population. ${ }^{22}$ Further, there is currently a growing interest in the role of various molecular markers in the treatment paradigm; ;,5,9,15 to our knowledge, however, the specific question of whether a better cognitive outcome is correlated to a favorable genetic profile has not been previously addressed.

Approximately one-quarter of patients with LGGs report serious problems with their neurocognitive functioning, particularly memory concentration, ${ }^{34}$ language, and executive functions, ${ }^{38,40,43,48}$ including at presentation. ${ }^{23}$ Previous studies have suggested that many patients with LGG suffer from neurocognitive deficits and compromised health-related quality of life (HRQoL), ${ }^{1,32}$ These deficits are historically considered to be location dependent, as it is known that frontal lobe tumors cause deficits including executive function, decision making, memory, and emotion, whereas dominant temporal lesions affect language and occipital lesions cause visuospatial deficits. ${ }^{10}$ Previously, only basic evaluations of cognitive skills were performed in brain tumor patients (i.e., the Mini-Mental State Examination or related tools). A comprehensive neurocognitive evaluation using a battery of tests is a far more sensitive method for detecting subtle cognitive changes and has become the standard protocol in many neurooncological centers.

The role of resection in LGG and its impact on cognition has not been thoroughly established. Further, the best surgical approach for maximal safe tumor resections, preoperative evaluation, and intraoperative techniques, including awake mapping and neuromonitoring, are still not common practice worldwide. This study addresses the long-term neurocognitive effects and outcome of surgery, with intraoperative awake mapping when appropriate, for LGG.

\section{Methods \\ Study Population}

We conducted a retrospective analysis of prospectively collected data from patients with LGG who underwent craniotomy for tumor removal at Tel Aviv Sourasky Medical Center between 2010 and 2014. A total of 192 patients were initially identified. Patients with infratentorial and intraventricular tumors, as well as those who only underwent needle or open biopsies and those who did not have complete pre- and postoperative cognitive evaluations, were excluded, leaving 49 patients with supratentorial, low-grade glial tumors and complete pre- and postoperative neurocognitive assessments, who were included in the study. The cohort is of nonconsecutive patient data due to patients' noncompliance with postoperative assessment or up-front refusal to consent. All patients included in the study underwent a comprehensive preoperative neurocognitive evaluation and follow-up evaluation at 3-28 months (mean 6.06 months) after surgery. We also performed a subgroup analysis of only those patients whose follow-up examinations were performed less than 12 months after surgery $(n=43$, mean follow-up 4.1 months, range 3-10 months). The 6 patients who were excluded from this subgroup analysis had a mean time to follow-up of 17.8 months. Side dominance was typically determined by handedness, with support from functional MRI (fMRI) data for patients undergoing awake craniotomies.

Patient-related and tumor-related variables are summarized in Table 1.

Molecular and histopathological data were gathered from routine pathology reports within patient files.

\section{Volumetric Analysis}

Tumor volumetrics were obtained from preoperative MRI as well as a follow-up MRI study that was performed within 4 weeks of the follow-up cognitive evaluation (either before or after) for all but 2 patients. For each patient, tumor volume was measured with T2-weighted FLAIR sequences; preoperative and follow-up images were coregistered to ensure maximal accuracy. The tumor volume was then segmented across all image slices and the region of interest was measured and computed using Brainlab navigation software (iPlan). Results are displayed in cubic centimeters. All measurements were manually performed by a single investigator (O.B.).

\section{Neurocognitive Tests}

Neurocognition was assessed utilizing a standardized test battery. The evaluation protocol included tests for estimated premorbid intelligence, attention and working memory, memory, learning, language, visuomotor organization, and executive functions. When available, alternate forms were used to minimize practice effects (Table 2). Cognitive functions and domains were grouped and analyzed similarly to previously published data by other groups. ${ }^{40,43,53}$ An expert neurosurgical staff neuropsychologist performed all tests.

Scores on each test were transformed into z-scores according to the mean and standard deviation of normative healthy control data for each specific test. For verbal tasks, Hebrew versions of tests and norms were used. ${ }^{20,54} \mathrm{~A}$ mean $\mathrm{z}$-score of tests included in a particular cognitive domain was calculated to represent the overall function level in that domain.

\section{Surgery}

All surgeries were conducted in a single institution by a single surgeon (Z.R.). Twenty-three of 26 operations in the dominant hemisphere were performed with intraoperative mapping for language, motor, and cognitive function in an awake setting. Our methods of awake craniotomy and mapping have been described elsewhere. ${ }^{12,28,29}$ Our method of intraoperative multisite direct cortical stimulation ${ }^{11}$ (i.e., applying simultaneous cortical stimulation at multiple locations) reveals additional language dysfunction when performed at sites that did not produce disruption when stimulation was applied at a single point. Functional regions identified by this technique are presumed to be significant to language circuitry and are spared during surgery.

\section{Pathology}

Immunohistochemistry for IDH1-R132H was done on 5- $\mu \mathrm{m}$-thick formalin-fixed, paraffin-embedded tumor sections. Antigen retrieval was performed in citrate buf- 
TABLE 1. Patient and tumor characteristics

\begin{tabular}{|c|c|}
\hline Characteristic & Value \\
\hline \multicolumn{2}{|l|}{ Patient characteristics } \\
\hline \multicolumn{2}{|l|}{ Sex } \\
\hline Male & 28 \\
\hline Female & 21 \\
\hline \multicolumn{2}{|l|}{ Age in yrs } \\
\hline Mean & 35.46 \\
\hline Range & $22-66$ \\
\hline \multicolumn{2}{|l|}{ Education in yrs } \\
\hline Mean & 14.52 \\
\hline Range & $11-19$ \\
\hline \multicolumn{2}{|l|}{ Hand dominance } \\
\hline Left & 44 \\
\hline Right & 5 \\
\hline \multicolumn{2}{|l|}{ Tumor characteristics } \\
\hline \multicolumn{2}{|l|}{ Diagnosis } \\
\hline Astrocytoma (WHO II) & 9 \\
\hline Oligodendroglioma (WHO II) & 25 \\
\hline PXA & 3 \\
\hline DNET & 2 \\
\hline Ganglioglioma & 3 \\
\hline Pilocytic astrocytoma (WHO I) & 2 \\
\hline Oligoastrocytoma (WHO II) & 5 \\
\hline \multicolumn{2}{|l|}{ Dominance } \\
\hline Dominant side & 26 \\
\hline Nondominant side & 23 \\
\hline \multicolumn{2}{|l|}{ Volume in $\mathrm{cm}^{3}$} \\
\hline Mean & 45.69 \\
\hline Range & $1.31-174.61$ \\
\hline \multicolumn{2}{|l|}{$\mathrm{IDH}^{*}$} \\
\hline Positive & 29 \\
\hline Negative & 19 \\
\hline \multicolumn{2}{|l|}{ p53† } \\
\hline Positive & 21 \\
\hline Negative & 20 \\
\hline \multicolumn{2}{|l|}{ GFAP* } \\
\hline Positive & 37 \\
\hline Negative & 11 \\
\hline \multicolumn{2}{|l|}{ Proliferation index (MIB/Ki-67) } \\
\hline Mean & $3.57 \%$ \\
\hline Range & $0-10 \%$ \\
\hline
\end{tabular}

DNET = dysembryoplastic neuroepithelial tumor; PXA = pleomorphic xanthoastrocytoma.

Values are numbers of patients or tumors unless otherwise indicated.

* Data were available for 48 patients.

$\dagger$ Data were available for 41 patients.

fer ( $\mathrm{pH}$ 6.0) in a microwave oven. An antibody specific for the mutant IDH1-R132H protein (H09, 1:100 dilution; Dianova) was used. A secondary antibody labeled with streptavidin conjugate (Dako/Agilent) was used as a de-
TABLE 2. Neuropsychological testing protocol

\begin{tabular}{|c|c|}
\hline $\begin{array}{l}\text { Cognitive Domain \& } \\
\text { Abilities Tested }\end{array}$ & Test \\
\hline \multicolumn{2}{|l|}{ Attention \& working memory } \\
\hline $\begin{array}{l}\text { Auditory attention \& working } \\
\text { memory }\end{array}$ & $\begin{array}{l}\text { WAIS-III Hebrew Digit Span } \\
\text { (forward \& backward) }\end{array}$ \\
\hline $\begin{array}{l}\text { Spatial attention \& working } \\
\text { memory }\end{array}$ & $\begin{array}{l}\text { WMS-III Spatial Span (forward \& } \\
\text { backward) }\end{array}$ \\
\hline \multicolumn{2}{|l|}{ Visuomotor organization } \\
\hline Preop visuomotor organization & Rey complex figure test-copy \\
\hline Postop visuomotor organization & Taylor alternative figure test-copy \\
\hline \multicolumn{2}{|l|}{ Memory \& learning } \\
\hline Preop visual long-term memory & $\begin{array}{l}\text { Rey complex figure test-delayed } \\
\text { memory }\end{array}$ \\
\hline $\begin{array}{l}\text { Postop visual long-term } \\
\text { memory }\end{array}$ & $\begin{array}{l}\text { Taylor alternative complex figure } \\
\text { test-delayed memory }\end{array}$ \\
\hline Auditory short-term memory & RAVLT (trials $1 \&$ 6) \\
\hline Auditory long-term memory & RAVLT (trial 8) \\
\hline Verbal learning & $\begin{array}{l}\text { RAVLT: total learning composite } \\
\text { score }\end{array}$ \\
\hline \multicolumn{2}{|l|}{ Language } \\
\hline Naming & Hebrew naming test \\
\hline Semantic fluency & COWAT: semantic fluency \\
\hline \multicolumn{2}{|l|}{ Executive function } \\
\hline Phonemic fluency & COWAT: phonemic fluency \\
\hline Response inhibition & Stroop test-interference score \\
\hline \multicolumn{2}{|l|}{ IQ predictor } \\
\hline Abstract thinking & WAIS-III Hebrew Similarities \\
\hline
\end{tabular}

COWAT = Controlled Oral Word Association Test; RAVLT = Rey Auditory Verbal Learning Test; WAIS-III = Wechsler Adult Intelligence Scale, Third Edition; WMS-III = Wechsler Memory Scale, Third Edition.

tection system. A senior pathologist evaluated the results of the immunostaining.

Immunohistochemistry for p53 and GFAP was done on 5- $\mu \mathrm{m}$-thick formalin-fixed, paraffin-embedded tumor sections. Antibodies specific for p53 (DO7, 453M96, 1:100 dilution; Cellmark) and GFAP (258R/16, 1:50 dilution; Cellmark) were used. A secondary antibody labeled with the streptavidin biotin kit (Universal) was used as a detection system (Dako). A senior pathologist evaluated the results of the immunostaining as part of the routine pathological report.

\section{Statistical Analysis}

Statistical analysis was performed using SPSS, version 17.0 (SPSS, Inc.). To examine the effect of surgery on a specific neurocognitive ability and on tumor volumetrics, we used a paired t-test. To identify the effect of a molecular marker on neurocognitive outcome, we used the Student t-test. We also present the $\mathrm{p}$ values obtained after correction for multiple comparisons (correction for family-wise error [FWE]). To identify the effect of resection on overall cognitive outcome, we used repeated-measures ANOVA. To examine the relationship between change in 
cognitive ability following surgery and extent of resection or residual tumor volume, we used Pearson's correlation. In all tests, a $p$ value of $<0.05$ was considered statistically significant.

Our institutional review board approved this study, and informed consent was obtained from participants.

\section{Results}

A total of 49 adult patients (28 male and 21 female) were included in this study. Their mean age at the time of surgery was 35.4 years (range 22-66 years). Patient and tumor variables are summarized in Table 1 . Tumor locations are shown in Fig. 1. The mean extent of resection was $79.4 \%$.

In all patients, the premorbid estimated intelligence and abstract thinking were within the normal range (i.e., less than $1 \mathrm{SD}$ from those of the population mean).

However, the baseline performance on cognitive tests showed the presence of cognitive deficits of varying severity at presentation. Memory was severely impaired in this population $(\mathrm{z}=-4.03)$. Near-significant impairments were noted in learning $(z=0.089)$, language $(z=0.72)$, naming $(\mathrm{z}=0.7)$, and delayed recall $(\mathrm{z}=0.9)$, and the performance in these areas was considered "low average." Subgroup analysis showed that patients who harbored tumors in the dominant hemisphere had lower scores (Rey Auditory Verbal Learning Test: total learning mean $\mathrm{z}=$ -1.0 , delayed recall mean $\mathrm{z}=-1.2$, naming mean $\mathrm{z}=-0.9$, phonemic fluency mean $\mathrm{z}=-0.8$ ). Thus, the mean performance of all patients as a group showed subtle impairment and was at the level of average and low average, whereas patients with dominant-side tumors showed significant cognitive impairment at presentation.

\section{Comparison of Preoperative Versus Follow-Up Cognitive Function \\ Overall Cohort}

Significant improvement following surgery was observed in memory ( $p<0.001$, uncorrected; $p<0.001$, cor- rected for FWE) and executive functions ( $p=0.031$, uncorrected; $p=0.124$, corrected for FWE). No significant improvement was noted regarding language, attention and working memory, or visuomotor organization (Fig. 2).

Although not all domains improved significantly, there was a trend of improvement across all domains. In a repeated-measures ANOVA model, postresection testing resulted in a significant beneficial effect for memory $(\mathrm{F}[1,26]=19.254, \mathrm{p}=0.000)$, while a trend was noted for executive functions $(\mathrm{F}[1,26]=3.311, \mathrm{p}=0.08)$.

No correlations were noted between cognitive changes and IDHI status, p53 or GFAP staining, or proliferation indices (Ki-67/MIB). Similarly, surgical parameters (i.e., extent of tumor resection [EOR] and residual tumor volume) were not significantly correlated with cognitive changes, although a trend of improvement was found with greater EOR and lower residual tumor volume.

The mean EOR for patients with p53-positive tumors was $84 \% \pm 16 \%$. There was a marginal correlation between p53 positivity and greater EOR $(\mathrm{p}=0.064)$ and a significant correlation between p53 positivity and better postoperative language function $(\mathrm{p}=0.005)$.

The subgroup analysis excluding patients who had a follow-up test more than 12 months after surgery did not alter the significance of the reported results.

\section{Patients With Dominant-Side Tumors}

Twenty-six patients harbored dominant-side tumors. The mean EOR in these cases was $76 \%$. Patients with dominant-side tumors experienced significant improvement in memory ( $p=0.001$, uncorrected; $p=0.004$, corrected for FWE), as well as in executive functions ( $\mathrm{p}=$ 0.016 , uncorrected; $p=0.064$, corrected for FWE), with no significant changes in other cognitive domains (Fig. 2). In a repeated-measures ANOVA model, postresection testing showed a significant beneficial effect on memory $(\mathrm{F}[1,14]=9.13, \mathrm{p}=0.009)$ and executive functions $(\mathrm{F}[1,14]$ $=6.23, \mathrm{p}=0.026$ ).

Extent of resection correlated nearly significantly $(\mathrm{p}=$ 0.06 ) with improvement in attention and working memory.

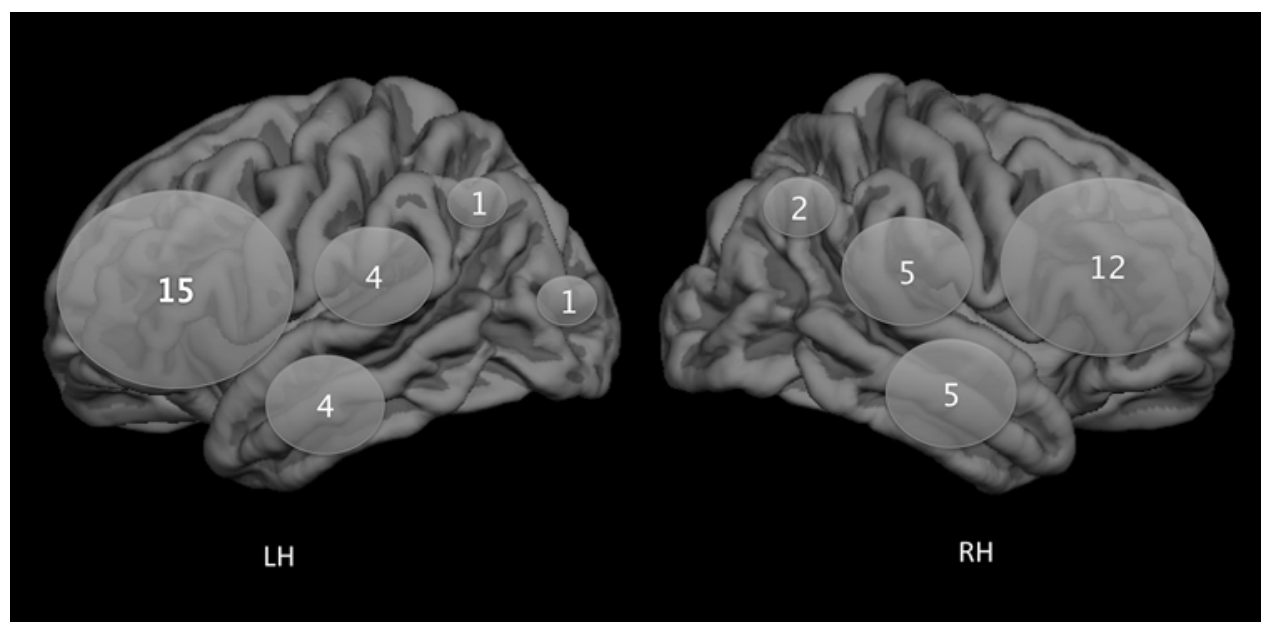

FIG. 1. Representation of tumor locations. Tumors are grouped according to their anatomical location and are presented on a generic brain (fsaverage5) surface representation imported from FreeSurfer (https://surfer.nmr.mgh.harvard.edu). LH = left hemisphere; $\mathrm{RH}=$ right hemisphere. 

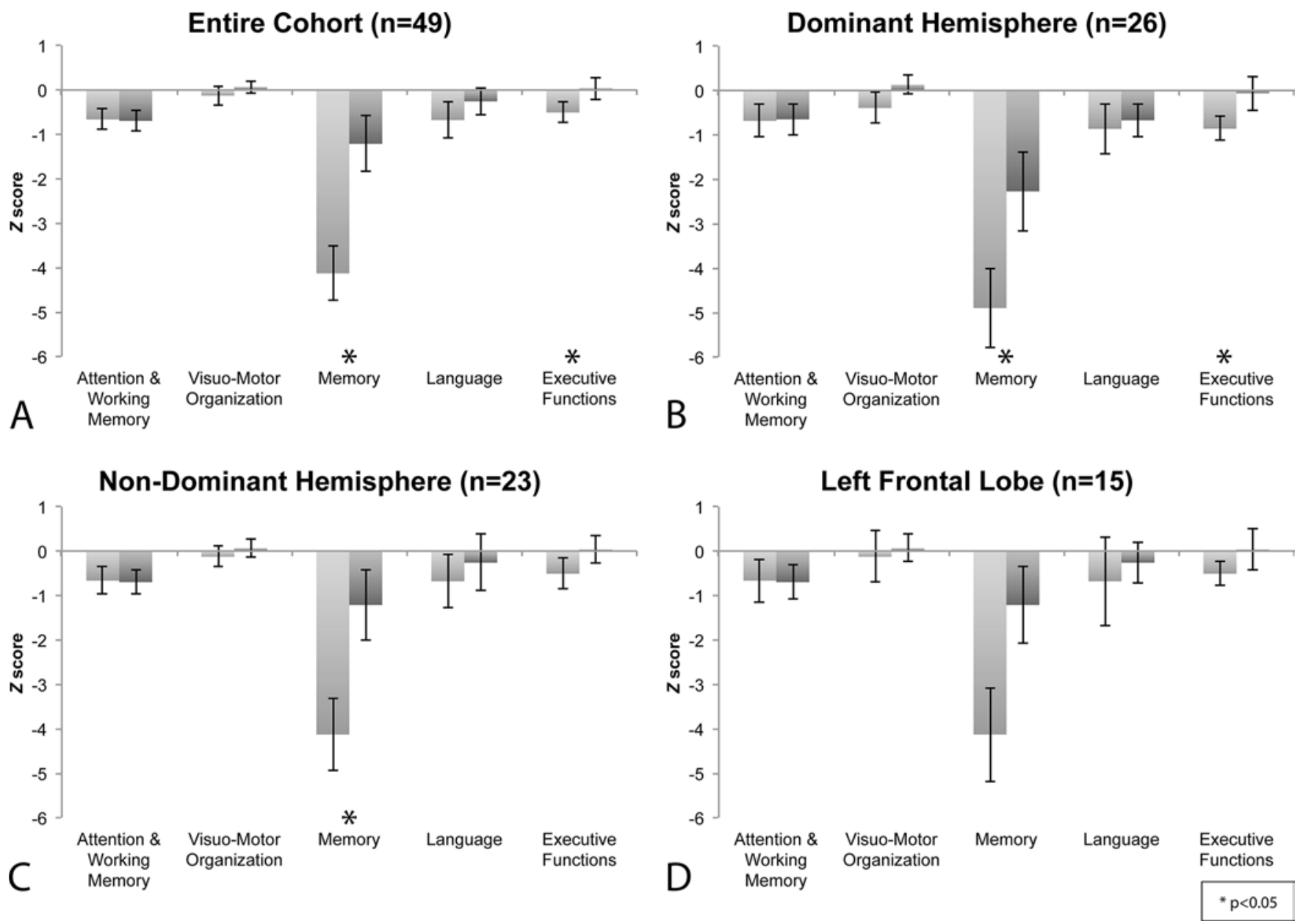

FIG. 2. Preoperative (light gray) and postoperative (dark gray) mean cognitive scores in LGG patients. A: Mixed cohort of patients with tumors in the dominant and nondominant side. B: Patients with dominant-side tumors. C: Patients with tumors in the nondominant side. D: Patients with tumors in the left frontal lobe. The expected population average score is 0 for each test. ${ }^{*} p<0.05$.

The mean EOR for patients with p53-positive tumors in this subgroup was $83 \% \pm 19 \%$; 553 positivity predisposed to a larger EOR $(\mathrm{p}=0.044)$ and was correlated with better postoperative language function $(\mathrm{p}=0.016)$. No other correlations were found between histopathological markers or surgical parameters (i.e., EOR, residual volume) and cognitive changes.

\section{Patients With Tumors on the Nondominant Side}

Twenty-three patients harbored nondominant-side tumors. Tumors varied significantly in size (1.84-141.45 $\mathrm{cm}^{3}$ ). The mean EOR for this subgroup was $83 \%$. After surgery, this group experienced significant improvement in the memory domain $(\mathrm{p}=0.001$, uncorrected; $\mathrm{p}=0.001$, corrected for FWE). A repeated-measures ANOVA model showed a significant beneficial effect only on memory $(\mathrm{F}[1,11]=2.27, \mathrm{p}=0.010)$ (Fig. 2).

IDH1 positivity was found in 13 of these 23 patients. IDH1 did not affect neurocognition but was associated with larger residual volume at follow-up $\left(9.42 \mathrm{vs} 2.41 \mathrm{~cm}^{3}\right.$, $\mathrm{p}=0.029$ ).

GFAP-positive tumors were found in 18 cases. GFAP positivity was associated with higher follow-up scores in attention and working memory tests $(\mathrm{p}=0.05)$. No correlations were found between p53 status or proliferation indices (Ki-67/MIB) and cognitive changes or surgical parameters.

\section{Patients With Left Frontal Tumors}

Fifteen patients presented with left frontal tumors. The extent of resection was $76.4 \%$. No statistically significant changes were found in any of the cognitive domains. A near-significant improvement was found in executive functions $(\mathrm{p}=0.059)($ Fig. 2).

Due to the small sample size in this group, histopathological and surgery-related correlations were not performed.

\section{Patients With Diffuse Versus Patients With Focal Tumors}

To evaluate the difference between "focal" tumors and more "diffuse" pathologies, the cognitive changes in patients with oligodendrogliomas $(\mathrm{n}=8)$ and patients with astrocytomas $(n=23)$ were evaluated. A significant beneficial effect of surgery on memory performance was observed in the oligodendroglioma group $(p=0.021)$. No 
other significant difference was evident between the 2 pathological groups.

\section{Discussion}

This study shows improvement in cognitive skills following resection of low-grade glial tumors as assessed by a battery of objective neurocognitive tests. Our data demonstrate improvement in memory and executive functions in a mixed patient cohort, as well as in a subpopulation of patients harboring dominant-side tumors. It should be noted that of 26 operations for dominant-side tumor resection, 23 were performed in an awake setting. The significant cognitive improvement in this subpopulation of patients emphasizes the importance of intraoperative mapping, when appropriate, for cognitive function preservation. We further attempted to associate cognitive changes after surgery with various markers, although more studies will be necessary to establish meaningful associations.

\section{Cognition in Brain Tumor Surgery}

Studies on the cognitive assessment of patients undergoing brain tumor surgery are limited. For patients with noninfiltrative tumors, cognitive improvement has been demonstrated after removal of meningiomas ${ }^{52}$ and arachnoid cysts. ${ }^{57}$ In patients with high-grade gliomas, poorer performance on cognitive tests is associated with shorter survival from the time of initial diagnosis, as well as from the time of tumor progression. ${ }^{19}$

LGG treatment is uniquely complex as the course of these lesions is usually indolent, and multiple treatment options are available. Through the long course of the disease, cognition is commonly impaired in this patient population. ${ }^{49}$ Cognitive changes can be attributed to the effects of both disease and treatment and may be caused by radiation, chemotherapy, seizures, medications, and surgery. ${ }^{25}$ A recent, comprehensive review described the scarcity of data available regarding the impact of surgery on cognition in glioma patients. ${ }^{42}$

Satoer et al. demonstrated that glioma surgery is possible without long-term damage to cognitive functions. ${ }^{41,43,44}$ Noll et al. showed that neurocognitive decline following resection of temporal lobe glioma is common, particularly in the domains of verbal memory and executive functioning, regardless of the hemisphere involved and occurring mostly in the subacute postsurgical period. ${ }^{27}$ In small patient series, several studies showed that surgery improved various functions and caused worsening of others, leaving the overall cognitive burden unchanged..$^{48,59}$ Verbal working memory has been shown to deteriorate immediately after surgery but then improve 3 months postoperatively to the baseline level (in 5 patients) or improve significantly above the preoperative status (in 3 other patients). ${ }^{50}$

Racine et al. conducted a retrospective analysis of 22 cases in which patients underwent awake craniotomies. Most of the patients presented with a decline in baseline cognitive functions, and 12 of them underwent follow-up cognitive examinations, which demonstrated no postoperative language decline; a decline in memory capabilities was, however, detected in follow-up assessment..$^{33}$

In our current study, 23 of 26 operations on dominant- side tumors were performed in an awake setting, and postoperative assessment showed improvement in memory and executive functions in these patients. Our study and that of Racine et al. both demonstrate the feasibility of cognitive function preservation in eloquent areas with use of awake mapping techniques when appropriate.

\section{Preoperative Cognitive Evaluation}

Our current study population consisted of well-educated patients with normal intelligence. Upon complete neurocognitive evaluation, subtle deficits that were overlooked in the regular neurological evaluation often surfaced. These subtle changes are important to recognize for both the patient and the treating physician, as cognitive changes assume a key role in treatment planning and decision making. Our data, along with other previously published data, ${ }^{33,43}$ emphasize the need for standardized neurocognitive assessment in all patients harboring intraaxial brain tumors.

\section{Memory}

Anterograde verbal memory decline is a common and potentially debilitating morbidity associated with temporal lobectomy (typically of the language-dominant side). ${ }^{17}$ Although memory has traditionally been attributed to the hippocampal region, fMRI experiments in normal subjects suggest that specific regions within the parietal cortex may also participate in episodic memory along with the hippocampal formation. ${ }^{55}$

Additional fMRI data emphasize the importance of neural networks, such as involvement of frontal networks in verbal learning, that may even appear useful in predicting postsurgical memory change in epilepsy surgery. ${ }^{47,51}$

When discussing memory as global capability, we combined both visual and auditory memory, along with learning capabilities (Table 2), demonstrating significant improvement in the postoperative cognitive assessment. Moreover, subgroup analysis of data from patients with dominant-side tumors, as well as from those with left frontal lobe tumors alone, demonstrated significant improvement in memory at follow-up, in contradiction to recently published results. ${ }^{33}$ Notably, verbal memory is typically associated with left-hemisphere function, whereas visual memory is typically associated with the right hemisphere. In this article, we discuss memory as a global capability, combining these 2 distinct capabilities.

Of note, a beneficial effect of surgery on memory performance was observed in the oligodendroglioma group when compared with the astrocytoma group $(p=0.021)$. This could reflect a resectability advantage with the more focal pathology compared with the more "diffuse" one. As the number of patients in this analysis is small $(n=8)$, this result should be validated in larger studies.

\section{Executive Functions}

Impairments of motor and executive function are the hallmark deficits associated with frontal lobe resection.7,26 Postoperative executive function decline following frontal lobe resection may include changes in attention and multitasking, fluency, response inhibition, concept formation, or problem solving. Executive dysfunction has also been described after anterior temporal resection for epilepsy, 
and in these cases, it is hypothesized to result from a disconnection between frontal and temporal lobe networks. ${ }^{2}$ Predictors of new or increased executive impairments after frontal lobe resection include intact presurgical executive functioning, ${ }^{14}$ continued seizures after surgery, ${ }^{14}$ and presurgical depressed mood. ${ }^{8}$

Our cohort included 27 patients with frontal lobe tumors, 15 of which were on the dominant side. Data from the entire cohort as well as from the subgroup with dominant-side tumors showed significant improvement in executive function following surgery. This improvement could be a direct result of reducing mass effect, or it could be due to subsequent resolution of peritumoral edema. Moreover, as LGGs are slow-growing tumors, the tumor-infiltrated areas may undergo plasticity and, possibly, are not functionally significant to the large-scale neural network responsible for cognitive functioning.

\section{Cognition and Histopathological Markers}

IDH1 Status. Isocitrate dehydrogenase 1 (IDH1) mutations are associated with improved survival in patients with $\mathrm{LGG},{ }^{58}$ as well as with possible better response to treatment in these patients. ${ }^{13,16}$ The role of IDH1 as a marker has recently been emphasized as new data demonstrate that most LGGs without an IDH mutation are molecularly and clinically similar to glioblastoma. ${ }^{3}$ Tumors in 29 patients in our cohort showed IDH1 positivity. Wefel et al. recently found that in malignant gliomas, patients with wild-type IDH1 show reduced neurocognitive function compared with those with mutant IDH1. ${ }^{56}$ A single association was made between IDH positivity and larger residual volume in a small subgroup of LGG patients whose tumors were in their nondominant hemisphere. The significance of this association could be a result of the sample size, but it could possibly point to a difference in resectability between IDH1-positive and IDH1-negative tumors.

p53. Loss of p53 tumor suppressor activity has been described as an early event in LGG formation and progression, ${ }^{30}$ and its prognostic role has remained controversial. ${ }^{24}$ In the present study, we found that in dominant-side tumors p53 positivity correlated with better postoperative language capabilities, as well as with greater extent of resection. Previously, p53 and other histological markers had not been shown to correlate with tumor resectability, ${ }^{7}$ and further studies are necessary to determine the significance of our current findings.

GFAP and Proliferation Indices. GFAP positivity was associated with higher follow-up scores in attention and working memory tests in patients with nondominant-side tumors. This association is likely a statistical observation, and its clinical significance remains to be determined.

No correlations were made between proliferation indices and cognitive change.

\section{Special Considerations}

All patients underwent a complete follow-up evaluation following surgery, and the postoperative data presented here are from the first postoperative follow-up visit. All but 6 patients were evaluated 3-10 months after surgery. This allows for adequate recovery time from surgery and reflects routine follow-up visits. There is some variability with the length of follow-up as 6 patients were evaluated more than a year after surgery, reflecting technical and compliance difficulties with these strenuous tests. These patients were kept in the analysis to avoid selection bias. As shown in a subgroup analysis, exclusion of the patients with delayed evaluation did not alter the reported results.

Our protocol for steroid treatment is quite standard, and in patients undergoing LGG resection, it includes a single intraoperative dose of $20 \mathrm{mg}$ dexamethasone and a rapid tapering schedule of postoperative steroid therapy, which is usually discontinued within 7-10 days of surgery. LGG patients were rarely considered for adjuvant therapies. None of the patients presented in this analysis had received adjuvant whole-brain radiation therapy between evaluations, and only 4 patients were treated with temozolomide prior to follow-up evaluation. Doses of other medications, such as antiepileptic drugs, could not be fully accounted for.

\section{Limitations}

We recognize the inherent flaws of this study associated with its retrospective nature.

The lack of a control group was inevitable in this analysis. A potential control group of patients who underwent biopsy only was excluded due to the fact that tumors not amenable to aggressive resection may represent a completely different and distinct disease entity. The nonconsecutive nature of this cohort represents a potential selection bias. Patients undergoing resection of intraaxial brain tumors at our institution routinely undergo full neuropsychological evaluations. A few patients were excluded due to their refusal to consent, and others did not have complete pre- and postoperative evaluations due to technical limitations.

Other potential confounding factors associated with cognitive impairment are vast, and accounting for all of them is nearly impossible.

\section{Conclusions}

Our study shows that surgery for removal of low-grade glial tumors is associated with cognitive improvement in memory and executive functions with no apparent evidence for deterioration in other cognitive domains. Preoperative evaluation, teamwork, patient selection, and liberal use of awake mapping techniques when appropriate are imperative in maintaining cognitive function in patients undergoing these challenging operations. New observations were made herein regarding p53 and GFAP positivity and their possible association with improved neurocognition and tumor resectability.

Evidence has been accumulating to support the notion that cognition results from the dynamic interactions of distributed brain areas operating in large-scale networks. ${ }^{4}$ The mechanisms by which cognitive improvement following tumor resection is possible remain unclear.

Early, aggressive resection of LGG has recently been advocated for improved survival. The improvement of cognitive outcome after surgery is another advantage that favors early resection in low-grade glial tumors. 


\section{References}

1. Aaronson NK, Taphoorn MJ, Heimans JJ, Postma TJ, Gundy CM, Beute GN, et al: Compromised health-related quality of life in patients with low-grade glioma. J Clin Oncol 29:4430-4435, 2011

2. Bell B, Lin JJ, Seidenberg M, Hermann B: The neurobiology of cognitive disorders in temporal lobe epilepsy. Nat Rev Neurol 7:154-164, 2011

3. Brat DJ, Verhaak RG, Aldape KD, Yung WK, Salama SR, Cooper LA, et al: Comprehensive, integrative genomic analysis of diffuse lower-grade gliomas. N Engl J Med 372:24812498, 2015

4. Bressler SL, Menon V: Large-scale brain networks in cognition: emerging methods and principles. Trends Cogn Sci 14:277-290, 2010

5. Chen R, Ravindra VM, Cohen AL, Jensen RL, Salzman KL, Prescot AP, et al: Molecular features assisting in diagnosis, surgery, and treatment decision making in low-grade gliomas. Neurosurg Focus 38(3):E2, 2015

6. Daniels TB, Brown PD, Felten SJ, Wu W, Buckner JC, Arusell RM, et al: Validation of EORTC prognostic factors for adults with low-grade glioma: a report using intergroup 8672-51. Int J Radiat Oncol Biol Phys 81:218-224, 2011

7. Dulay MF, Busch RM: Prediction of neuropsychological outcome after resection of temporal and extratemporal seizure foci. Neurosurg Focus 32(3):E4, 2012

8. Dulay MF, Busch RM, Chapin JS, Jehi L, Najm I: Executive functioning and depressed mood before and after unilateral frontal lobe resection for intractable epilepsy. Neuropsychologia 51:1370-1376, 2013

9. Eckel-Passow JE, Lachance DH, Molinaro AM, Walsh KM, Decker PA, Sicotte H, et al: Glioma groups based on 1p/19q, IDH, and TERT promoter mutations in tumors. N Engl J Med 372:2499-2508, 2015

10. Fang S, Wang Y, Jiang T: The influence of frontal lobe tumors and surgical treatment on advanced cognitive functions. World Neurosurg 91:340-346, 2016

11. Gonen T, Gazit T, Korn A, Kirschner A, Perry D, Hendler T, et al: Intra-operative multi-site stimulation: Expanding methodology for cortical brain mapping of language functions. PLoS One 12:e0180740, 2017

12. Grossman R, Nossek E, Sitt R, Hayat D, Shahar T, Barzilai O, et al: Outcome of elderly patients undergoing awake-craniotomy for tumor resection. Ann Surg Oncol 20:1722-1728, 2013

13. Hartmann C, Hentschel B, Tatagiba M, Schramm J, Schnell O, Seidel C, et al: Molecular markers in low-grade gliomas: predictive or prognostic? Clin Cancer Res 17:4588-4599, 2011

14. Helmstaedter C, Gleissner U, Zentner J, Elger CE: Neuropsychological consequences of epilepsy surgery in frontal lobe epilepsy. Neuropsychologia 36:681-689, 1998

15. Hottinger AF, Hegi ME, Baumert BG: Current management of low-grade gliomas. Curr Opin Neurol 29:782-788, 2016

16. Houillier C, Wang X, Kaloshi G, Mokhtari K, Guillevin R, Laffaire J, et al: IDH1 or IDH2 mutations predict longer survival and response to temozolomide in low-grade gliomas. Neurology 75:1560-1566, 2010

17. Ivnik RJ, Sharbrough FW, Laws ER Jr: Effects of anterior temporal lobectomy on cognitive function. J Clin Psychol 43:128-137, 1987

18. Jakola AS, Myrmel KS, Kloster R, Torp SH, Lindal S, Unsgård G, et al: Comparison of a strategy favoring early surgical resection vs a strategy favoring watchful waiting in lowgrade gliomas. JAMA 308:1881-1888, 2012

19. Johnson DR, Sawyer AM, Meyers CA, O'Neill BP, Wefel JS: Early measures of cognitive function predict survival in patients with newly diagnosed glioblastoma. Neuro Oncol 14:808-816, 2012
20. Kavé G: Standardization and norms for a Hebrew naming test. Brain Lang 92:204-211, 2005

21. Klein M: Neurocognitive functioning in adult WHO grade II gliomas: impact of old and new treatment modalities. Neuro Oncol 14 (Suppl 4):iv17-iv24, 2012

22. Klein M, Duffau H, De Witt Hamer PC: Cognition and resective surgery for diffuse infiltrative glioma: an overview. J Neurooncol 108:309-318, 2012

23. Klein M, Heimans JJ, Aaronson NK, van der Ploeg HM, Grit J, Muller M, et al: Effect of radiotherapy and other treatment-related factors on mid-term to long-term cognitive sequelae in low-grade gliomas: a comparative study. Lancet 360:1361-1368, 2002

24. Levidou G, El-Habr E, Saetta AA, Bamias C, Katsouyanni $\mathrm{K}$, Patsouris E, et al: P53 immunoexpression as a prognostic marker for human astrocytomas: a meta-analysis and review of the literature. J Neurooncol 100:363-371, 2010 (Erratum in $\mathbf{J}$ Neurooncol 100:373, 2010)

25. McAleer MF, Brown PD: Neurocognitive function following therapy for low-grade gliomas. Semin Radiat Oncol 25:210218, 2015

26. Mesulam MM: From sensation to cognition. Brain 121:1013-1052, 1998

27. Noll KR, Weinberg JS, Ziu M, Benveniste RJ, Suki D, Wefel JS: Neurocognitive changes associated with surgical resection of left and right temporal lobe glioma. Neurosurgery 77:777-785, 2015

28. Nossek E, Matot I, Shahar T, Barzilai O, Rapoport Y, Gonen $\mathrm{T}$, et al: Intraoperative seizures during awake craniotomy: incidence and consequences: analysis of 477 patients. Neurosurgery 73:135-140, 2013

29. Nossek E, Matot I, Shahar T, Barzilai O, Rapoport Y, Gonen $T$, et al: Failed awake craniotomy: a retrospective analysis in 424 patients undergoing craniotomy for brain tumor. J Neurosurg 118:243-249, 2013

30. Ohgaki H, Kleihues P: Genetic profile of astrocytic and oligodendroglial gliomas. Brain Tumor Pathol 28:177-183, 2011

31. Pan IW, Ferguson SD, Lam S: Patient and treatment factors associated with survival among adult glioblastoma patients: a USA population-based study from 2000-2010. J Clin Neurosci 22:1575-1581, 2015

32. Pelletier G, Verhoef MJ, Khatri N, Hagen N: Quality of life in brain tumor patients: the relative contributions of depression, fatigue, emotional distress, and existential issues. J Neurooncol 57:41-49, 2002

33. Racine CA, Li J, Molinaro AM, Butowski N, Berger MS: Neurocognitive function in newly diagnosed low-grade glioma patients undergoing surgical resection with awake mapping techniques. Neurosurgery 77:371-379, 2015

34. Reijneveld JC, Sitskoorn MM, Klein M, Nuyen J, Taphoorn MJ: Cognitive status and quality of life in patients with suspected versus proven low-grade gliomas. Neurology 56:618623, 2001

35. Ringel F, Pape H, Sabel M, Krex D, Bock HC, Misch M, et al: Clinical benefit from resection of recurrent glioblastomas: results of a multicenter study including 503 patients with recurrent glioblastomas undergoing surgical resection. Neuro Oncol 18:96-104, 2016

36. Riva M, Bello L: Low-grade glioma management: a contemporary surgical approach. Curr Opin Oncol 26:615-621, 2014

37. Sanai N, Berger MS: Extent of resection influences outcomes for patients with gliomas. Rev Neurol (Paris) 167:648-654, 2011

38. Sanai N, Chang S, Berger MS: Low-grade gliomas in adults. J Neurosurg 115:948-965, 2011

39. Sanai N, Polley MY, McDermott MW, Parsa AT, Berger MS: An extent of resection threshold for newly diagnosed glioblastomas. J Neurosurg 115:3-8, 2011 
40. Santini B, Talacchi A, Squintani G, Casagrande F, Capasso R, Miceli G: Cognitive outcome after awake surgery for tumors in language areas. J Neurooncol 108:319-326, 2012

41. Satoer D, Vincent A, Smits M, Dirven C, Visch-Brink E: Spontaneous speech of patients with gliomas in eloquent areas before and early after surgery. Acta Neurochir (Wien) 155:685-692, 2013

42. Satoer D, Visch-Brink E, Dirven C, Vincent A: Glioma surgery in eloquent areas: can we preserve cognition? Acta Neurochir (Wien) 158:35-50, 2016

43. Satoer D, Visch-Brink E, Smits M, Kloet A, Looman C, Dirven C, et al: Long-term evaluation of cognition after glioma surgery in eloquent areas. J Neurooncol 116:153-160, 2014

44. Satoer D, Vork J, Visch-Brink E, Smits M, Dirven C, Vincent A: Cognitive functioning early after surgery of gliomas in eloquent areas. J Neurosurg 117:831-838, 2012

45. Schwamm LH, Van Dyke C, Kiernan RJ, Merrin EL, Mueller J: The Neurobehavioral Cognitive Status Examination: comparison with the Cognitive Capacity Screening Examination and the Mini-Mental State Examination in a neurosurgical population. Ann Intern Med 107:486-491, 1987

46. Shields LB, Choucair AK: Management of low-grade gliomas: a review of patient-perceived quality of life and neurocognitive outcome. World Neurosurg 82:e299-e309, 2014

47. Sidhu MK, Stretton J, Winston GP, Symms M, Thompson PJ, Koepp MJ, et al: Memory fMRI predicts verbal memory decline after anterior temporal lobe resection. Neurology 84:1512-1519, 2015

48. Talacchi A, Santini B, Savazzi S, Gerosa M: Cognitive effects of tumour and surgical treatment in glioma patients. J Neurooncol 103:541-549, 2011

49. Taphoorn MJB: Neurocognitive sequelae in the treatment of low-grade gliomas. Semin Oncol 30 (6 Suppl 19):45-48, 2003

50. Teixidor P, Gatignol P, Leroy M, Masuet-Aumatell C, Capelle L, Duffau H: Assessment of verbal working memory before and after surgery for low-grade glioma. J Neurooncol 81:305-313, 2007

51. Trenerry MR, Meador KJ: Predicting memory change after temporal lobectomy for epilepsy. Neurology 84:1508-1509, 2015

52. Tucha O, Smely C, Preier M, Becker G, Paul GM, Lange KW: Preoperative and postoperative cognitive functioning in patients with frontal meningiomas. J Neurosurg 98:21-31, 2003

53. Tucha O, Smely C, Preier M, Lange KW: Cognitive deficits before treatment among patients with brain tumors. Neurosurgery 47:324-334, 2000

54. Vakil E, Greenstein Y, Blachstein H: Normative data for composite scores for children and adults derived from the Rey Auditory Verbal Learning Test. Clin Neuropsychol 24:662-677, 2010

55. Vincent JL, Snyder AZ, Fox MD, Shannon BJ, Andrews JR, Raichle ME, et al: Coherent spontaneous activity identifies a hippocampal-parietal memory network. J Neurophysiol 96:3517-3531, 2006

56. Wefel JS, Noll KR, Rao G, Cahill DP: Neurocognitive function varies by IDH1 genetic mutation status in patients with malignant glioma prior to surgical resection. Neuro Oncol 18:1656-1663, 2016

57. Wester K, Hugdahl K: Arachnoid cysts of the left temporal fossa: impaired preoperative cognition and postoperative improvement. J Neurol Neurosurg Psychiatry 59:293-298, 1995

58. Wrensch M, McMillan A, Wiencke J, Wiemels J, Kelsey K, Patoka J, et al: Nonsynonymous coding single-nucleotide polymorphisms spanning the genome in relation to glioblastoma survival and age at diagnosis. Clin Cancer Res 13:197-205, 2007

59. Yoshii Y, Tominaga D, Sugimoto K, Tsuchida Y, Hyodo A, Yonaha $\mathrm{H}$, et al: Cognitive function of patients with brain tumor in pre- and postoperative stage. Surg Neurol 69:51-61, 2008

\section{Disclosures}

The authors report no conflict of interest concerning the materials or methods used in this study or the findings specified in this paper.

\section{Author Contributions}

Conception and design: Ram, Barzilai. Acquisition of data: all authors. Analysis and interpretation of data: Ram, Barzilai, Ben Moshe, Shofty. Drafting the article: Barzilai. Critically revising the article: Ram, Barzilai. Reviewed submitted version of manuscript: all authors. Statistical analysis: Shofty. Administrative/ technical/material support: Ram, Barzilai. Study supervision: Ram.

\section{Correspondence}

Zvi Ram: Tel Aviv Medical Center, Tel Aviv, Israel. zviram@ tlvmc.gov.il. 